\title{
SUBSTREAM ALLOCATION IN LAYERED P2P STREAMING
}

\author{
Yifeng He, Ivan Lee, and Ling Guan \\ Department of Electrical and Computer Engineering, \\ Ryerson University, Toronto, Ontario, M5B 2K3, Canada
}

\begin{abstract}
In layered P2P streaming system, how to allocate number of the copies for each layer is a challenging problem. In this paper, we present a substream allocation scheme in layered P2P streaming. The proposed allocation scheme is adaptive to the request rate and number of the qualified peers. The simulation results show that the proposed allocation scheme enables the system to achieve an overall better quality compared to the general allocation schemes with fixed allocation percentages. In addition, the proposed allocation scheme can accelerate the growth of peer population in the initial stage of hybrid $\mathrm{P} 2 \mathrm{P}$ streaming systems.
\end{abstract}

\section{INTRODUCTION}

Peer-to-peer (P2P) streaming has attracted considerable attention in the recent years. In P2P streaming, peers communicate directly with each other for sharing and exchanging data as well as other resources such as storage. It is typical that several serving peers collectively stream the requested content to the receiving peer, since a single serving peer may not be able or willing to contribute an upload bandwidth enough for the media playback at the receiver. This multipoint-to-point communication can provide a higher overall throughput to the receiver, hence resulting in a higher quality [1].

Recently, the coordination mechanisms in multipointto-point P2P streaming have been reported in Peerstreaming [4] and PALS [5]. Peer selection and peer switching are studied in [6]. Tu et al. [2] presents an analytical framework to study the capacity growth in the hybrid streaming system, considering the unpredictable departure/failure of peers.

In layered coded P2P streaming, the video is encoded into multiple layers. A layer won't be able to be decoded if the lower layers are not available. The bit stream in layer $i$ is defined as substream $i$. After a streaming session is finished, the receiving peer will replicate one or multiple substreams, and become a qualified peer [2], which may serve other requesting peers in the next streaming session.

One of the major challenges in the layered P2P systems is how to allocate different number of the substreams for different layer. The lower layers have more importance than the higher layers. Therefore, more replicas should be made to increase the availability of the lower layers. The substream allocation is implemented by substream replication in the pool of qualified peers. A good allocation scheme can help the system to provide an overall good quality to the users, and accelerate the growth of the peer population in the $\mathrm{P} 2 \mathrm{P}$ systems.

The impact on the system performance with different allocation schemes has been studied in [3]. In this paper, we propose a novel allocation scheme in layered P2P streaming systems. Compared with the general allocation schemes with fixed allocation percentages [3], the proposed scheme can provide the users with a better quality. We also study how the proposed allocation scheme can accelerate the growth of the peer population in the initial stage of hybrid P2P streaming.

\section{SUBSTREAM ALLOCATION}

\subsection{Single-file system}

We first study a layered P2P streaming system with only a single video $k$ where $k$ is the video ID. In this subsection, we would like to determine how many copies of each substream should be stored among the pool of the qualified peers in order to achieve an overall high quality, if the total number of the online qualified peers $N_{k}$ is given.

The mean duration of the video is $L$. We encode the video into $M$ layers using layered video codec. Each layer has an average bit rate $B_{r}$. We assume that each substream is delivered from a peer via an independent path. The probability of successfully receiving substream $i$ is denoted as $q_{k i}$.

In our model, a central server manages the qualified peers and controls the substream replication. Each request is first sent to the central server, which then selects a 
qualified peer to deliver substream $i(i=1,2, \ldots M)$ to the receiver, respectively.

We assume that each qualified peer is homogeneous in contributed storage and upload bandwidth. Each qualified peer can store only one substream and upload only one substream to the receiver. Each qualified peer alternates between "on" and "off" states. We model the "on" time as an exponentially distributed random variable with mean $T_{\text {on }}$, and the "off" time as another exponentially distributed random variable with mean $T_{\text {off }}$ [3]. The steady probability that a peer stays at the "on" state is given by $u=T_{o n} /\left(T_{o n}+T_{o f f}\right)$. There are totally $W_{k}$ qualified peers for video $k$ in the pool. The expected number of the online qualified peers is $N_{k}=u W_{k}$. We will next determine the number $N_{k i}$ of substream $i(i=1,2, \ldots M)$ in the pool in order to achieve a high expected quality.

The expected quality is related to the request rate and the number of the substream for each layer. The request to the video is modeled as a Poisson process with a rate $\lambda_{k}$. The qualified peers with substream $i$ are trunked together to serve the requests for substream $i$. A request will be rejected without waiting in a queue, if there is no corresponding substream available in the serving pool. The steady probability $P_{B k i}$ that a request for substream $i$ is rejected can be found from Erlang $B$ trunking model $[2,7]$ :

$$
P_{B k i}=\frac{\left(\lambda_{k} L\right)^{N_{k i}} \frac{1}{\left(N_{k i}\right) !}}{\sum_{n=0}^{N_{k i}}\left[\left(\lambda_{k} L\right)^{n} \frac{1}{n !}\right]}
$$

We assume that the session will be finished successfully once its request is accepted. The probability of receiving substream $i$ can be given by $q_{k i}=1-P_{B k i}$. The probability $P_{k m}$ that the user receives the video with $m$ layers can be written as

$$
P_{k m}=\left\{\begin{array}{lc}
1-q_{k 1} & m=0, \\
\left(1-q_{k(m+1)}\right) \prod_{i=1}^{m} q_{k i} & m=1,2, \ldots(M-1), \\
\prod_{i=1}^{M} q_{k i} & m=M
\end{array}\right.
$$

The expected distortion of the received video is given by

$$
E\left(D_{k M}\right)=\sum_{m=0}^{M}\left(P_{k m} D_{k m}\right)
$$

where $D_{k m}$ is the average distortion of received video with $m$ decodable layers.

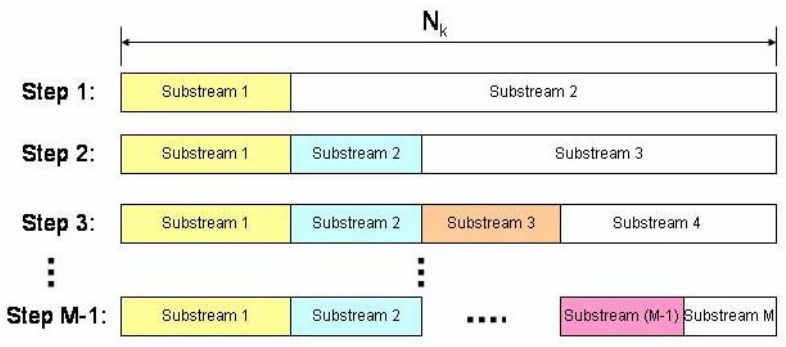

Fig. 1. Illustration of the proposed substream allocation scheme

The proposed substream allocation scheme is illustrated in Fig. 1. The expected number of the online qualified peers $N_{k}$ is equal to the number of the substreams stored in the qualified peers, since each peer stores only one substream in our model. We determine the number of substream $i$ in a stepwise way. In step 1, we divide $N_{k}$ substreams into two kinds: substream 1 and substream 2 . We then find the number $N_{k 1}$ * of substream 1 by exhaustive search in $N_{k}$ in order to minimize the expected distortion $E\left(D_{k 2}\right)$, the expected distortion of received video with at most 2 layers. Generally, in the step $i$ $(i=2,3, \ldots(M-1))$, given $N_{k j} *(j=1,2, \ldots(i-1))$, we divide $N_{k}$ into $(i+1)$ layers and search within the number of left substreams $N_{L}(i)=N_{k}-\left(N_{k 1} *+N_{k 2} *+\ldots+N_{k(i-1)} *\right)$ to find $N_{k i} *$ in order to

$$
\begin{aligned}
& \min E\left(D_{k(i+1)}\right)=\sum_{m=0}^{i+1}\left(P_{k m} D_{k m}\right) \\
& \text { s.t. } \quad \sum_{j=1}^{i+1} N_{k j}^{*}=N_{k}
\end{aligned}
$$

In the step $(M-1)$, we can obtain $N_{k(M-1)} *$. The left substreams are assigned to layer $M: N_{k M} *=N_{k}-\left(N_{k 1} *+\right.$ $\left.N_{k 2} *+\ldots+N_{k(M-1)} *\right)$. Finally, we obtain the number of the substreams for each of the $M$ layers, which can be denoted with an allocation vector $V_{k}=\left\{N_{k 1} *, N_{k 2} *, \ldots N_{k M} *\right\}$.

The proposed scheme allocates the number of the copies for each substream by adapting to the request rate and the number of total substreams. The central server carries out the substream allocation by controlling the substream replication in each qualified peer, so that all the substreams are placed following the allocation vector $V_{k}$.

\subsection{Multi-file system}

We now extend the analysis to a simplified multi-file system with $F$ files. Each file has an average length of $L$. We divide the multi-file system into $F$ virtual sub-systems, each of which deals with only one file.

The popularity of video $k(k=1,2, \ldots F)$ is modeled as a random variable following $\operatorname{Zipf}$ distribution $f_{Z}(k)$. The aggregate request rate is $\lambda_{T}$. The request rate to video $k$ is $\lambda_{k}=\lambda_{T} f_{Z}(k)$. There are totally $N_{T}$ online qualified peers in 
the system, we allocate $N_{k}$ qualified peers to store video $k$ based on its popularity. Therefore we have $N_{k}=N_{T} f_{Z}(k)$.

Given the number of the online qualified peers for video $k$, we then can further allocate the substreams for each layer of video $k$ using the proposed allocation scheme. The substream allocation vector for video $k$ can be obtained as $V_{k}=\left\{N_{k 1} *, N_{k 2} *, \ldots N_{k M} *\right\}$.

The average distortion of the $F$ videos in the simplified multi-file system is given by

$$
D_{T}=\sum_{k=1}^{F}\left(f_{Z}(k) E\left(D_{k M}\right)\right)
$$

where $E\left(D_{k M}\right)$ is the expected distortion of video $k$ with at most $M$ layers.

\section{EVOLUTION OF QUALITY AND POPULATION UNDER THE PROPOSED ALLOCATION SCHEME}

In this section, we study how the expected video quality and peer population evolve under the proposed substream allocation scheme in the hybrid P2P streaming. In hybrid $\mathrm{P} 2 \mathrm{P}$ system, a newly released video file is first pushed to the central server. In the initial stage, the central server cooperates with the qualified peers to serve streams to the users since there are less qualified peers available [2].

In our model, each qualified peer stores and uploads only one substream. We assume the Poisson request rate is fixed at $\lambda_{H}$. The length of the video is $L$. We analyze the population of the qualified peers in a discrete-time manner. Each interval has a length of $L$. If $G$ streaming sessions are initiated in the $n^{\text {th }}(n=1,2, \ldots)$ interval and completed successfully, these $G$ receivers will become new qualified peers in the $(n+1)^{\text {th }}$ interval [2].

The server has a fixed upload bandwidth, which can deliver $S$ substreams. The population of the qualified peers in the $n^{\text {th }}$ interval is denoted as $Z(n)$, which is equal to the number of the substreams in the $n^{\text {th }}$ interval since each qualified peer uploads one substream. Under the proposed allocation scheme, the total $(S+Z(n))$ substreams are deployed as $V_{H}(n)=\left\{N_{1}(n), N_{2}(n), \ldots N_{M}(n)\right\}$, where $M$ is the number of the layers, $N_{i}(n)(i=1,2, \ldots M)$ is the number of the substream $i$ in the $n^{\text {th }}$ interval. Given the allocated number of each substream and the request rate $\lambda_{H}$, the expected distortion of the received video in the $n^{\text {th }}$ interval can be calculated from equation (3).

In the $(n+1)^{t h}$ interval, the expected number of newly generated qualified peers $Y_{m}(n+1)$ with $m$ decodable layers is given by

$$
Y_{m}(n+1)=\lambda_{H} L P_{m}(n) \quad m=1,2, \ldots M
$$

where $P_{m}(n)$ is the probability that a peer receives a video with $m$ decodable layers in the $n^{\text {th }}$ interval. The population of the qualified peers in $(n+1)^{\text {th }}$ interval is given by $Z(n+1)=Z(n)+\left(Y_{1}(n+1)+Y_{2}(n+1)+\ldots+Y_{M}(n+1)\right)$. Under the proposed allocation scheme, the total $(S+Z(n+1))$ substreams in the $(n+1)^{\text {th }}$ interval are deployed as $V_{H}(n+1)=\left\{N_{1}(n+1), N_{2}(n+1), \ldots N_{M}(n+1)\right\}$. Then the expected distortion of the received video in the $(n+1)^{t h}$ interval can also be calculated from equation (3).

In this way, we can get the evolution of the expected quality and expected population of the qualified peers under the proposed allocation scheme.

\section{SIMULATIONS}

We encode the "Akiyo" CIF sequence into 6 layers using SNR-scalable extension of H.264/AVC [8]. Each layer has an average bit rate of $25 \mathrm{Kbps}$. The total average bit rate of the video is $150 \mathrm{Kbps}$. Each group of pictures (GOP) consists of 16 frames.

There are 20 videos in the multi-file P2P system. The popularity of the video follows Zipf distribution with parameter $\alpha=1.1$. The number of the requests follows Poisson process with a rate $\lambda$ ranging from 0.001 to 0.25 requests/s. The average duration of the video is 1 hour.

Each peer contributes an upload bandwidth of $30 \mathrm{Kbps}$, and an average storage of 15 Mbytes. The mean "on" time of the peer is 9 hours, and the mean "off" time is 1 hour. Therefore, the availability of the peer is 0.9 .

We compare the proposed allocation scheme to three general allocation schemes [3], in which the number of the copies $C_{m}$ of layer $m(m=1,2, \ldots, M)$ is given by $C_{m}=a(M-m+1)^{b}$, where $a$ is a normalization constant, $b$ is $0.5,1.0$, or 2.0 , respectively [3].

Fig. 2 shows the average PSNR versus request rate under different allocation schemes. The number of the qualified peers is 1000 . The general schemes allocate the channels with fixed percentages without adapting to the change of the request rates, therefore the expected quality drops dramatically when the request rate is large. On the other hand, the proposed scheme adapts to the request rate, thus achieving a higher quality.

Fig. 3 shows the average PSNR with different number of the qualified peers when the request rate is fixed at 0.1 requests/s. When the number of the qualified peers is small, the proposed allocation scheme allocates a larger percentage of the substreams to the lower-layers, thus increasing the quality quickly, while the general schemes don't allocate the substreams in an efficient way, thus lowering the quality.

The evolution of expected quality and the number of the qualified peers in the initial stage of hybrid P2P streaming system is evaluated in Fig. 4 and Fig. 5, respectively. The upload bandwidth that the server uses to serve this video is $5 \mathrm{Mbps}$, which can support 200 concurrent substreams. The request rate is 0.1 requests/s. We don't consider peer-failure in this scenario. Fig. 4 shows average quality evolution with time. In the beginning, the proposed scheme allocates more lowerlayer substreams to reduce the number of the rejected 
requests. Once the number of lower-layer substreams is large enough, the system will increase the number of higher-layer substreams to enhance the quality. In the initial stage, the general schemes allocate some higherlayer substreams. However, most of these high-layer substreams cannot be decoded since the availability of the lower-layer substreams is low. The similar impact can be seen on the peer population growth shown in Fig. 5. The proposed scheme allocates the substreams in a better way, so that it can generate more qualified peers.

\section{CONCLUSIONS}

In this paper, we presented a substream allocation scheme in layered P2P streaming. The number of the substreams for each layer is determined by a stepwise approach. The proposed allocation scheme is adaptive to the request rate and the number of the available qualified peers. The proposed allocation scheme enables the layered P2P system to achieve an overall better quality compared to the general allocation schemes with fixed allocation percentages. In addition, the proposed allocation scheme can accelerate the growth of the video quality and peer population in the initial stage of hybrid P2P streaming systems.

In the future, we are going to investigate the system performance considering peer heterogeneity and peer failure.

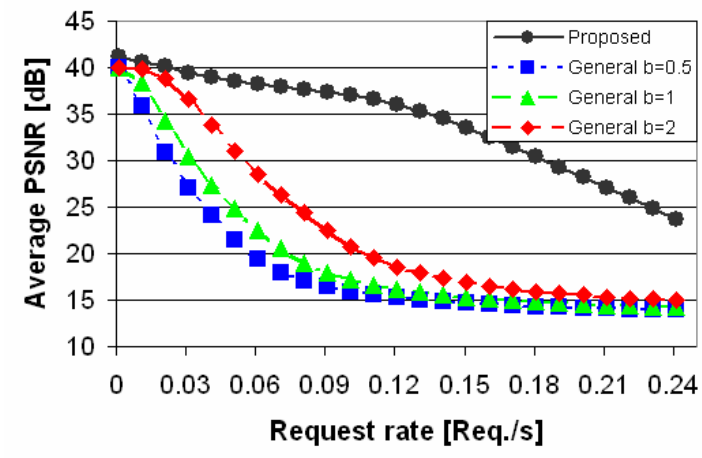

Fig.2. Average PSNR with varying request rate

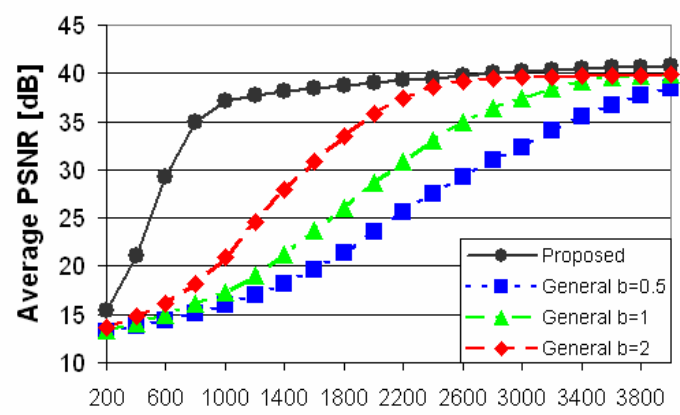

Num. of Qua. Peers

Fig.3. Average PSNR with varying number of qualified peers

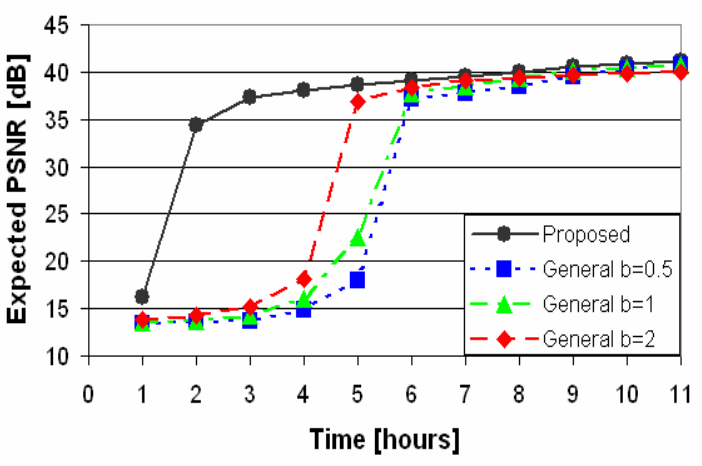

Fig.4. The evolution of expected PSNR

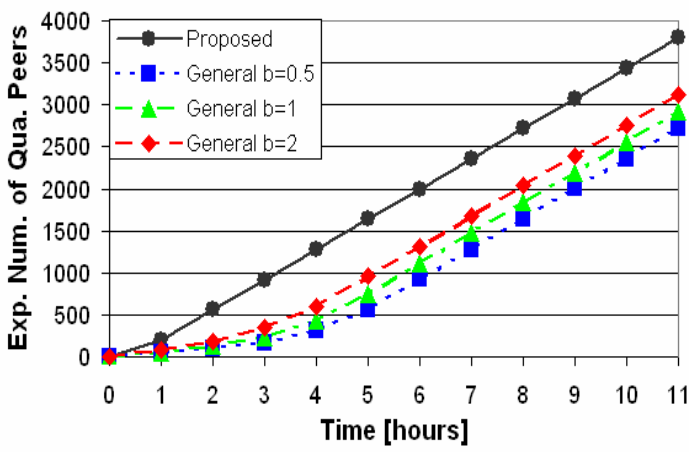

Fig.5. The evolution of expected number of qualified peers

\section{REFERENCES}

[1] D. Xu, M. Hefeeda, S. Hambrusch, and B. Bhargava, "On peer-to-peer media streaming", in Proc. of IEEE ICDCS, pp. 363-371, Jul. 2002.

[2] Y. Tu, J. Sun, M. Hefeeda, Y. Xia, S. Prabhakar, "An Analytical Study of Peer-to-Peer Media Streaming Systems", ACM Transactions on Multimedia Computing, Communications, and Applications, Accepted, Aug. 2005.

[3] Y. Shen, Z. Liu, S. P. Panwar, K. W. Ross and Y. Wang, "Streaming Layered Encoded Video using Peers", in Proc. of IEEE ICME, pp. 966-969, Jul. 2005.

[4] J. Li, "PeerStreaming: A practical receiver-driven peer-topeer media streaming system", Microsoft Technical Report (MSR-TR-2004-101), Sep. 2004.

[5] R. Rejaie and A. Ortega, "PALS: Peer to peer adaptive layered streaming," in Proc. of ACM NOSSDAV, pp.153-161, June 2003.

[6] M. Hefeeda, A. Habib, B. Botev, D. Xu, and B. Bhargava, "PROMISE: peer-to-peer media streaming using collectcast," in Proc. of ACM Multimedia, pp. 45-54, Nov. 2003.

[7] T. S. Rappaport, Wireless Communications: Principles and Practice, 2nd edition, Prentice Hall, 2002

[8] H. Schwarz, D. Marpe, and T. Wiegand, "SNR-scalable extension of H.264/AVC", in Proc. of IEEE ICIP, Vol. 5, pp. 3113-3116, Oct. 2004. 